\title{
Auricularia auricular-judae polysaccharide attenuates lipopolysaccharide-induced acute lung injury by inhibiting oxidative stress and inflammation
}

\author{
LI ZHUAN-YUN $^{1 *}$, YAO XUE-PING ${ }^{2 *}$, LIU BIN $^{1}$, HA NIZAIER REHEMAN ${ }^{1}$, \\ $\mathrm{GAO}_{\mathrm{YANG}}{ }^{1}$, $\mathrm{SUN}_{\mathrm{ZHAN}}{ }^{2}$ and $\mathrm{MA} \mathrm{QI}^{2}$ \\ ${ }^{1}$ Clinical Medical College, ${ }^{2}$ Laboratory of Functional Experiment, Preclinical Medicine \\ College, Xinjiang Medical University, Urumqi, Xinjiang 830011, P.R. China
}

Received March 24, 2015; Accepted May 5, 2015

DOI: $10.3892 /$ br. 2015.470

\begin{abstract}
Auricularia auricular-judae polysaccharide (AAP) has shown a variety of pharmacological properties. In the present study, the role of AAP in acute lung injury (ALI) induced by lipopolysaccharide (LPS) was analyzed in rats to further explore the possible underlying mechanisms. Adult Sprague-Dawley rats were randomly assigned into the control, AAP, LPS and LPS plus AAP groups. Rats were injected with LPS (10 mg/kg, intraperitoneal) to induce ALI. Rats in the LPS plus AAP group were treated with AAP for 7 days before the induction of ALI. The protein concentration in the bronchoalveolar lavage fluid (BALF) was measured. The animal lung edema degree was evaluated by the wet/dry (W/D) weight ratio. The myeloperoxidase (MPO) activity and malondialdehyde (MDA) level were assayed by MPO and MDA kits, respectively. The levels of inflammatory mediators, tumor necrosis factor- $\alpha$ (TNF- $\alpha$ ) and interleukin (IL)-6, were assayed by the enzyme-linked immunosorbent assay method. Pathological changes of lung tissues were observed by hematoxylin and eosin staining. The data showed that treatment with AAP significantly improved LPS-induced lung pathological changes, attenuated protein concentration in the BALF, inhibited MPO activity and reduced the MDA level and lung W/D weight ratio. AAP also inhibited the release of
\end{abstract}

Correspondence to: Mrs. Sun Zhan or Mrs. Ma Qi, Laboratory of Functional Experiment, Preclinical Medicine College, Xinjiang Medical University, 393 Xinyi Road, Urumqi, Xinjiang 830011, P.R. China

E-mail: sunzhan724@126.com

E-mail: maqi1111@126.com

*Contributed equally

Key words: acute lung injury, lipopolysaccharide, Auricularia auricular-judae polysaccharide, oxidative stress, inflammation
TNF- $\alpha$ and IL-6 in blood. The results indicated that AAP has a protective effect on LPS-induced ALI in rats.

\section{Introduction}

Acute respiratory distress syndrome (ARDS), an indication of acute lung injury (ALI), is highly associated with sepsis, multiple transfusions and trauma (1). ARDS is an inflammatory condition, manifested by a diffused alveolar damage, formation of hyaline membranes, protein-rich edema fluid in the alveolar spaces, capillary injury and disruption of the alveolar epithelium (2). Lipopolysaccharides (LPS) are thought to play a major role in initiating the inflammatory processes that result in ALI, mainly by dysfunction of the pulmonary surfactants (3). Exposure of LPS, derived from the cell wall of Gram-negative bacteria, is a well-known method to introduce acute lung inflammation and ARDS. LPS activates alveolar macrophages and causes neutrophils to infiltrate and damage the lungs.

Numerous studies have suggested that oxidant injury to the pulmonary microvasculature is an important mechanism in the pathogenesis of ARDS $(4,5)$. There are animal models to prove that pretreatment with scavengers of reactive oxygen species significantly reduces the pulmonary hypertension, hypoxia and increased microvascular permeability to proteins that otherwise characterizes ALI following infusion of endotoxin (6).

Auricularia auricular-judae is a medicinal edible fungus, which belongs to the basidiomycotina fungi, mainly distributed in China, Taiwan, Thailand and Indonesia. The fruit of Auricularia auricular-judae is rich in hetero-polysaccharides that consist of a D-glucose residue backbone with various chains of $\beta$-1,3-branch residues, such as mannose, glucose, xylose and glucuronic acid. It has been proved that black fungus polysaccharide not only exhibits an extremely high nutritional value, but also has various pharmacological functions in humans and animals. Black fungus polysaccharide has antioxidant $(7,8)$, blood lipid lowering $(9)$, antitumor $(10,11)$ and anti-radiation (12) activity. Therefore, the present study aimed to determine whether pretreatment of AAP on LPS-induced ARDS in rats could ameliorate the ALI. 


\section{Materials and methods}

Chemicals and reagents. LPS (Escherichia coli LPS, 055:B5) was purchased from Sigma Aldrich (St. Louis, MO, USA). The myeloperoxidase (MPO) and malondialdehyde (MDA) kits were purchased from Jiancheng Bioengineering Institute of Nanjing (Nanjing, China). Tumor necrosis factor- $\alpha$ (TNF- $\alpha$ ) and interleukin (IL)-6 ELISA kits were purchased from USCN Life Science Inc. (Wuhan, China). The fruit body of Auricularia auricular-judae was cultured in the Daxinganling region, Heilongjiang province, China.

Extraction of AAP. AAP was extracted by hot water and ultrasonic-assisted extraction. The concentrated supernatants were subsequently precipitated with 3 volumes of absolute ethanol (95\%) and maintained at $4^{\circ} \mathrm{C}$ overnight. The resulting precipitate was separated by centrifugation, dissolved in deionized water and subsequently dialyzed. The non-dialyzed portion was, in addition, lyophilized to result in a crude polysaccharide extract. The AAP was decolorized by hydrogen peroxide (13).

Animals and modeling. Adult Sprague-Dawley rats provided by the Laboratory Animal Center of Xinjiang Medical University (Xinjiang, China) were used in all the experiments. All the animal care and experimental procedures were approved by the Animal Care Committee of Xinjiang Medical University. Adult Sprague-Dawley rats were randomly assigned into the control, AAP, LPS and LPS plus AAP groups. The control animals received an equal volume of normal saline at the same time. The LPS group was induced by intraperitoneal injection of $10 \mathrm{mg} / \mathrm{kg}$ LPS. Rats in the LPS plus AAP group were treated with AAP for 7 days before LPS administration. Post-LPS infusion (12 h), animals were sacrificed by overdose of ethyl carbamate and blood samples were collected from the abdominal aorta. Blood samples were anticoagulated with EDTA and centrifuged at $3,000 \mathrm{x} \mathrm{g}$ for $10 \mathrm{~min}$ at $4^{\circ} \mathrm{C}$ and the plasma was stored at $-20^{\circ} \mathrm{C}$ until measurements were performed. Following death at the end of the protocol, the left lung was lavaged using $500 \mu \mathrm{l}$ of saline 3 times (total volume, $1.5 \mathrm{ml}$ ) for protein leakage. The right lung tissues were divided into 3 pieces, one immersed in $10 \%$ formalin solution for histopathological examination, one frozen in liquid nitrogen for quantitative analysis and the remaining part for measurement of the wet/dry (W/D) weight ratio.

Bronchoalveolar lavage. The lungs were lavaged with $500 \mu \mathrm{l}$ of saline 3 times (total volume, $1.5 \mathrm{ml}$ ). Retrieval volume was maximized by compression of the thorax following the last lavage. The protein concentration was determined using a protein kit.

Lung W/D weight ratio. The trachea and esophagus were separated from the lungs by blunt dissection and the wet weight of the latter was determined. Subsequently, the lungs were incubated at $60^{\circ} \mathrm{C}$ for 3-4 days to remove all moisture, the dry weight was measured and the ratio of wet-to-dry weight calculated.

MPO and MDA activities in lung homogenates. The MPO and MDA activities in the lung tissue were assayed by MPO and MDA kits, respectively, following the manufacturer's instructions.

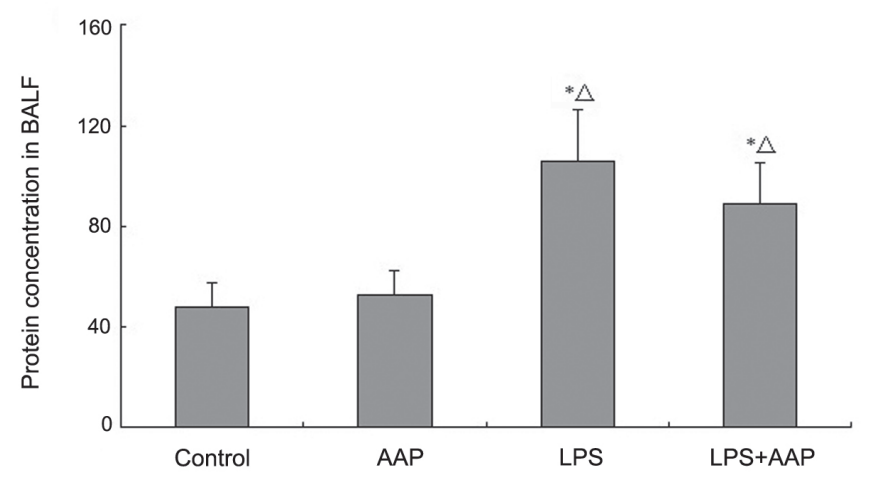

Figure 1. Effects of Auricularia auricular-judae polysaccharide (AAP) on the protein concentration in bronchoalveolar lavage fluid (BALF) of lipopolysaccharide (LPS)-induced acute lung injury rats. ${ }^{*} \mathrm{P}<0.05$ compared with the control, ${ }^{\triangle} \mathrm{P}<0.05$ compared with the AAP group.

Cytokines in blood. TNF- $\alpha$ and IL- 6 in blood samples were determined with ELISA kits from USCN Life Science Inc., performed according to the manufacturer's instructions. All the measurements were performed in duplicate.

Histological assessment. A section of the right lung was removed and put into $10 \%$ formaldehyde solution followed by dehydration, paraffin embedding, sectioning and hematoxylin and eosin staining sequentially. The changes of pathology were observed.

Statistical analysis. Results are presented as mean \pm standard deviation. For tests of significance between the groups, one-way analysis of variance was performed. Comparisons between two groups were performed using unpaired Student's t-test. $\mathrm{P}<0.05$ was considered to indicate a statistically significant difference. All the data were performed in $\geq 3$ independent experiments.

\section{Results}

Effects of AAP on protein concentration in bronchoalveolar lavage fluid $(B A L F)$. To confirm the efficacy of LPS exposure, the protein concentration in BALF was observed. As shown in Fig. 1, protein concentration in BALF markedly increased in the LPS group compared with the control and AAP groups. However, pretreatment with AAP caused the protein concentration in BALF to decrease compared with the LPS group.

Effects of AAP on lung W/D weight ratio. To investigate the effect of AAP on LPS-induced lung edema, W/D weight ratios were detected. As shown in Fig. 2, there were no significant differences between the control and AAP groups, which indicated that AAP had no effect on lung edema in normal rats. LPS injected for $12 \mathrm{~h}$ caused a significant increase in the lung W/D weight ratio compared with the control group $(\mathrm{P}<0.01)$. As shown in Fig. 3, in the AAP pretreated group the lung W/D weight ratios decreased compared with the LPS groups.

Effect of AAP on MDA level and MPO activity in lung tissues of LPS-treated rats. To assess the lung neutrophil burden within pulmonary tissues, lung MPO activity was measured. 


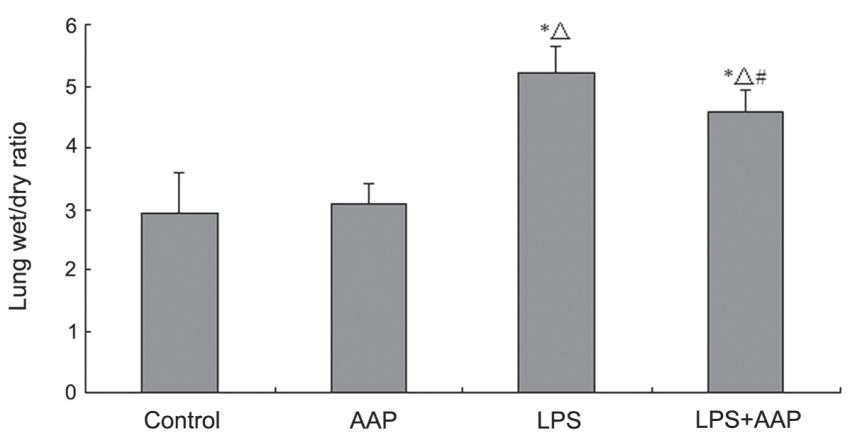

Figure 2. Effect of Auricularia auricular-judae polysaccharide (AAP) on the lung wet/dry ratio of lipopolysaccharide (LPS)-induced acute lung injury rats. ${ }^{*} \mathrm{P}<0.05$ compared with the control, ${ }^{\triangle \mathrm{P}}<0.05$ compared with the AAP group, ${ }^{*} \mathrm{P}<0.05$ compared with the LPS group.
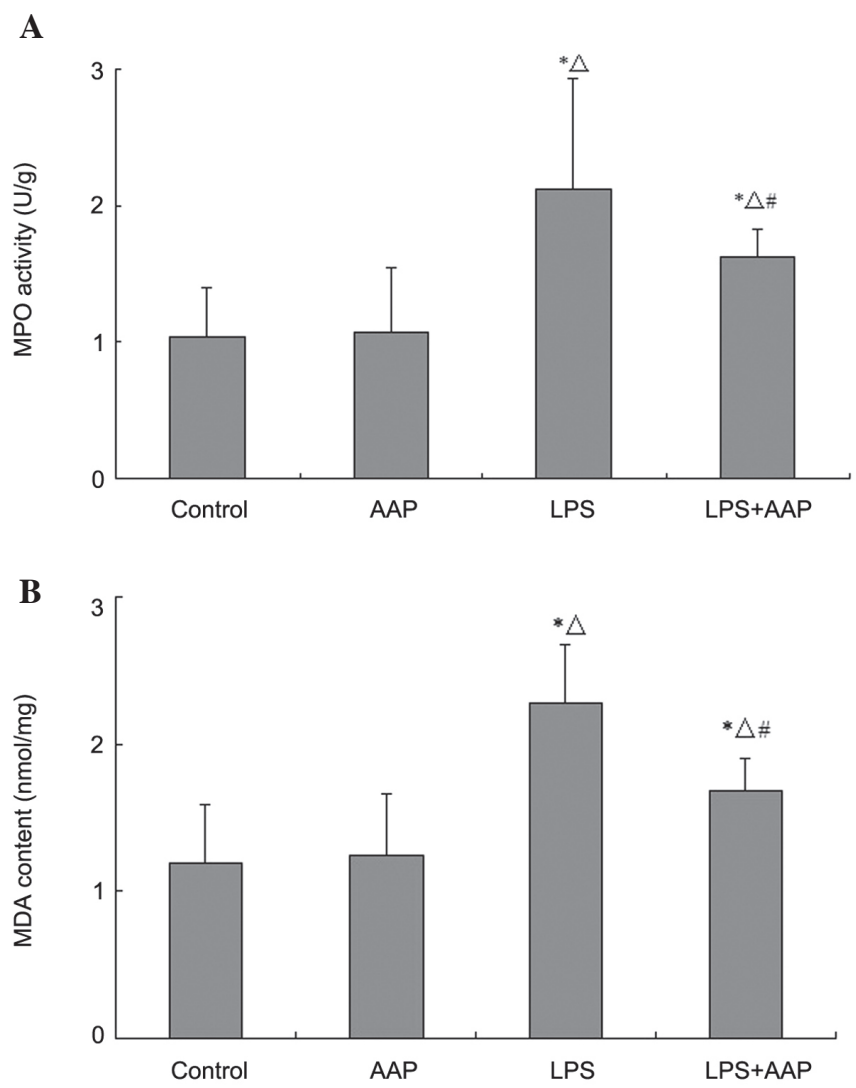

Figure 3. Effects of Auricularia auricular-judae polysaccharide (AAP) on the (A) myeloperoxidase (MPO) activity and (B) malondialdehyde (MDA) content in the lungs of lipopolysaccharide (LPS)-induced acute lung injury rats. ${ }^{\prime} \mathrm{P}<0.05$ compared with the control, ${ }^{\triangle} \mathrm{P}<0.05$ compared with the AAP group, ${ }^{,} \mathrm{P}<0.05$ compared with the LPS group.

As shown in Fig. 3, MPO activity increased significantly compared with the control and AAP groups, however, the MPO activity deceased in the LPS plus AAP group. In addition, LPS induced an increase in the MDA level in lung tissues and AAP significantly inhibited the MDA level.

Effect of AAP on TNF- $\alpha$ and IL-6 in the blood of LPS-treated rats. The concentration of TNF- $\alpha$ and IL- 6 in the blood represents pro-inflammatory mediators, which were thought to play crucial roles in the development of ALI. As shown in Fig. 4,
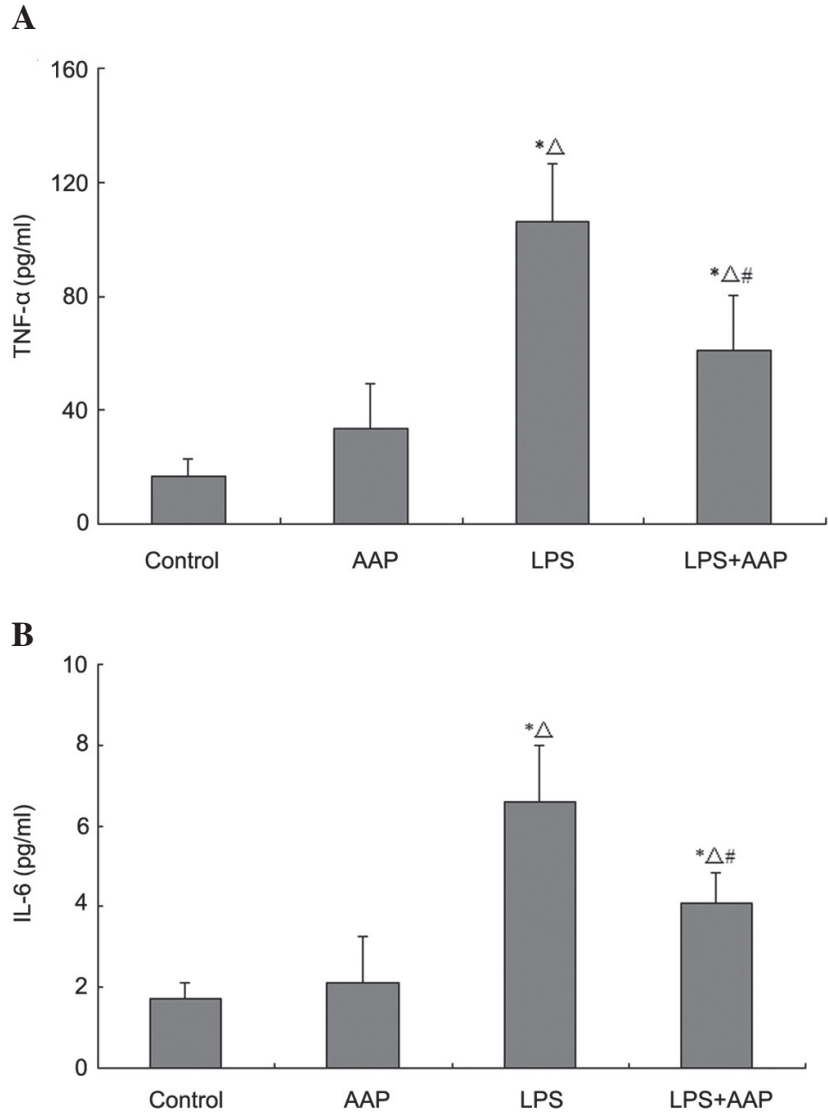

Figure 4. Effect of Auricularia auricular-judae polysaccharide (AAP) on the (A) tumor necrosis factor- $\alpha$ (TNF- $\alpha$ ) and (B) interleukin (IL)-6 levels in the blood of lipopolysaccharide (LPS)-induced rats. ${ }^{*} \mathrm{P}<0.05$ compared with the control, ${ }^{\triangle} \mathrm{P}<0.05$ compared with the AAP group, ${ }^{\#} \mathrm{P}<0.05$ compared with the LPS group.

TNF- $\alpha$ and IL- 6 levels increased markedly in the LPS group compared with the control and AAP groups, whereas these levels were decreased by AAP pretreatment.

Effect of AAP on LPS-mediated lung histopathological changes. In order to study the effects of AAP on ALI, the histological changes were determined following AAP treatment in LPS-treated rats. As shown in Fig. 5, in the control and heparin groups, lung tissue showed a normal structure and clear pulmonary alveoli under a light microscope. The changes in the LPS group, such as a large number of neutrophil sequestration and infiltration around the pulmonary vessel and airway, distributed in the alveolar and interstitial were observed. The LPS group pretreated with AAP markedly alleviated the LPS-induced pathological changes of the lung. These results indicated that AAP could protect rats from LPS-induced lung damage.

\section{Discussion}

ARDS is a life-threatening respiratory failure due to lung injury from a variety of precipitants (14). Despite advances in supportive treatment, mainly associated with protective ventilation (15) and a fluid conservative strategy, the morbidity and mortality of patients remains high (16). The lung pathogenesis of ALI/ARDS is characterized by the diffuse alveolar damage, 
A

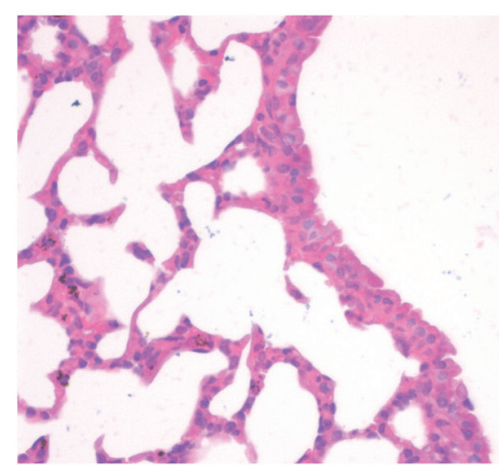

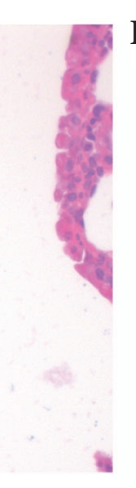

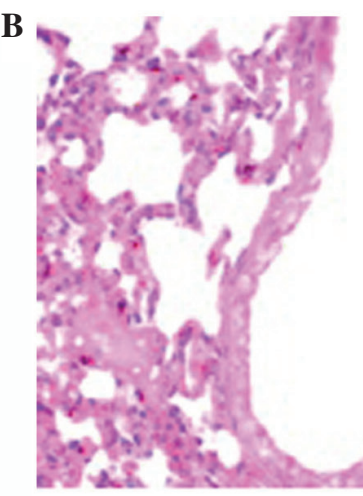

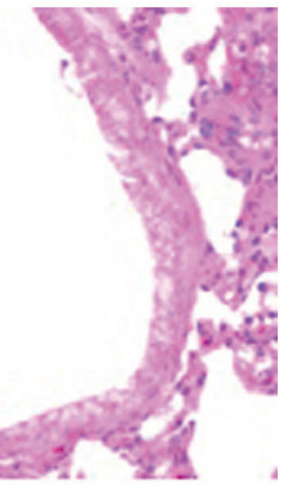

$\mathbf{D}$

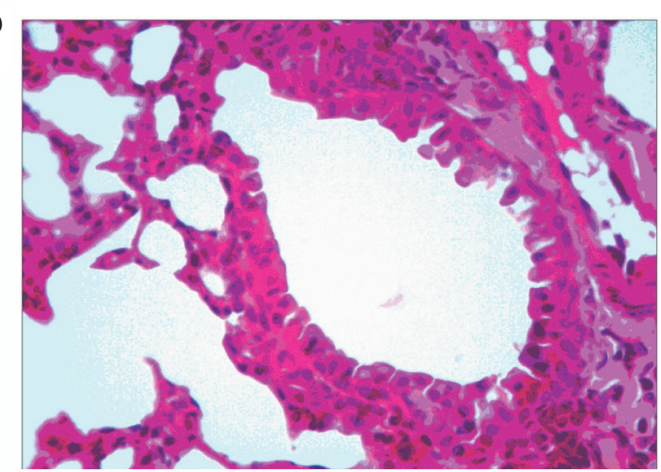

Figure 5. Pathological changes of lung tissues observed by hematoxylin and eosin staining (light microscopy; magnificaion, x200). (A) Control, (B) Auricularia auricular-judae polysaccharide (AAP), (C) lipopolysaccharide (LPS) and (D) LPS plus AAP groups.

alveolar capillary leakage and protein-rich pulmonary edema leading to the clinical manifestation of poor lung compliance, severe hypoxemia and bilateral infiltrates on chest radiograph (17). Neutrophils play a critical role in the pathogenesis of ALI/ARDS and when activated release harmful mediators, including cytokines, proteases, reactive oxygen species and matrix metalloproteinases, leading to further damage (18). Numerous studies have suggested that oxidative stress is an important mechanism in the pathogenesis of ARDS (4,5). In a previous study, pretreatment with scavengers of reactive oxygen species in an animal model has been shown to significantly reduce the damage, such as pulmonary hypertension, hypoxia and increased microvascular permeability, to protein (19).

LPS is a principal component of the outer membrane of Gram-negative bacteria and can enter the blood stream and elicit inflammatory responses that may lead to shock and ultimately to death (20). LPS-induced lung injury in the rat is frequently used as a model for studying ALI (21). Thus, this model was used in the present study to investigate the prevention of AAP on LPS-induced ALI in mice.

AAP has shown a variety of pharmacological properties. Black fungus polysaccharide has antioxidant $(7,8)$, blood lipid lowering (9), antitumor $(10,11)$ and anti-radiation (12) activity. In our previous study, we proved that AAP has anti-inflammation (22) and antioxidant functions (23). MPO is an enzyme located mainly in the primary granules of neutrophils and its main function is to kill microorganisms, but under certain conditions, it produces excess oxidants leading to tissue damage (24). In the present study, we found that MPO activity increased significantly following LPS administration. By contrast, pretreatment of AAP significantly decreased MPO activity and reduced neutrophil infiltration. MDA is a lipid peroxidation marker used to assess lipid peroxidation due to increased oxidative stress (25). The blood levels of MDA were markedly increased in LPS-induced mice, which could be significantly reversed by the pretreatment of AAP. The lung W/D weight ratio was evaluated as an index of pulmonary edema. It was found that AAP decreased the LPS-induced lung W/D ratio. The protein concentration in BALF markedly increased in the LPS group. However, following pretreatment with AAP, the protein concentration in BALF decreased compared with the LPS group. These results suggested that AAP has a protective effect on LPS-induced ALI.

Excessive cytokine-mediated inflammation was thought to play crucial roles in the development of ALI. In the present study, LPS caused a significant increase in the level of TNF- $\alpha$ and IL-6 in blood compared with the control group. By contrast, AAP treatment significantly reduced TNF- $\alpha$ and IL-6 secretion. These results suggested that the protective effects of AAP on LPS-induced ALI are partly attributed to inhibition of TNF- $\alpha$ and IL- 6 production.

In conclusion, the present study demonstrated that AAP significantly ameliorated the lung injury induced by LPS in rats via the inhibition of pro-inflammatory cytokine expression and antioxidation. These results may provide a theoretical foundation for treating ALI in the future.

\section{Acknowledgements}

The present study was supported by the National Natural Science Foundation of China (grant no. 81260454) and the 
Students Research Training Program of Xinjiang Medical University (grant no. CX2014021).

\section{References}

1. Frutos-Vivar F, Ferguson ND and Esteban A: Epidemiology of acute lung injury and acute respiratory distress syndrome. Semin Respir Crit Care Med 27: 327-336, 2006.

2. Matuschak GM and Lechner AJ: Acute lung injury and the acute respiratory distress syndrome: Pathophysiology and treatment. Mo Med 107: 252-258, 2010.

3. Kaplan RL, Sahn SA and Petty TL: Incidence and outcome of the respiratory distress syndrome in gram-negative sepsis. Arch Intern Med 139: 867-869, 1979.

4. Cross CE, Forte T, Stocker R, Louie S, Yamamoto Y, Ames BN and Frei B: Oxidative stress and abnormal cholesterol metabolism in patients with adult respiratory distress syndrome. J Lab Clin Med 115: 396-404, 1990.

5. Sabarirajan J, Vijayaraj P and Nachiappan V: Induction of acute respiratory distress syndrome in rats by lipopolysaccharide and its effect on oxidative stress and antioxidant status in lung. Indian J Biochem Biophys 47: 278-284, 2010.

6. Blackwell TS, Blackwell TR, Holden EP, Christman BW and Christman JW: In vivo antioxidant treatment suppresses nuclear factor-kappa B activation and neutrophilic lung inflammation. J Immunol 157: 1630-1637, 1996.

7. Ma H, Xu X and Feng L: Responses of antioxidant defenses and membrane damage to drought stress in fruit bodies of Auricularia auricula-judae. World J Microbiol Biotechnol 30: 119-124, 2014.

8. Xu L, Ma Q, Yao XP, et al: The effect of extract from black funguson liver and small intestine's MDA, GSH-Px in the septic shock rats. J XinJiang Med Univ 36: 450-455, 2013 (In Chinese)

9. Han CR and Xu LP: Black fungus polysaccharide extraction, purification and antihypelipidemic effect. J Chin Inst Food Sci Technol 15: 54-58, 2007 (In Chinese).

10. Reza MA, Hossain MA, Lee SJ, Yohannes SB, Damte D, Rhee MH, Jo WS, Suh JW and Park SC: Dichlormethane extract of the jelly ear mushroom Auricularia auricula-judae (higher Basidiomycetes) inhibits tumor cell growth in vitro. Int J Med Mushrooms 16: 37-47, 2014.

11. Cai R, Zhu XS, Qu HY, et al: Extraction of Black Fungus Polysaccharide and Its Inhibitory Effect on Glioma. Acta Med Univ Sci Technol Huazhong 42: 578-581, 2013 (In Chinese).

12. Bai H, Wang Z, Cui J, Yun K, Zhang H, Liu RH, Fan Z and Cheng C: Synergistic radiation protective effect of purified Auricularia auricular-judae polysaccharide (AAP IV) with grape seed procyanidins. Molecules 19: 20675-20694, 2014.
13. Zhang H, Wang ZY, Yang L, Yang X, Wang X and Zhang Z: In vitro antioxidant activities of sulfated derivatives of polysaccharides extracted from Auricularia auricular. Int J Mol Sci 12: 3288-3302, 2011

14. Dushianthan A, Grocott MP, Postle AD and Cusack R: Acute respiratory distress syndrome and acute lung injury. Postgrad Med J 87: 612-622, 2011.

15. Delong P, Murray JA and Cook CK: Mechanical ventilation in the management of acute respiratory distress syndrome. Semin Dial 19: 517-524, 2006.

16. Buregeya E, Fowler RA, Talmor DS, Twagirumugabe T, Kiviri W and Riviello ED: Acute respiratory distress syndrome in the global context. Glob Heart 9: 289-295, 2014.

17. Gattinoni L, Bombino M, Pelosi P, Lissoni A, Pesenti A, Fumagalli $\mathrm{R}$ and Tagliabue M: Lung structure and function in different stages of severe adult respiratory distress syndrome. JAMA 271: 1772-1779, 1994.

18. Zemans RL, Colgan SP and Downey GP: Transepithelial migration of neutrophils: Mechanisms and implications for acute lung injury. Am J Respir Cell Mol Biol 40: 519-535, 2009.

19. Weng TI, Wu HY, Kuo CW and Liu SH: Honokiol rescues sepsis-associated acute lung injury and lethality via the inhibition of oxidative stress and inflammation. Intensive Care Med 37: 533-541, 2011.

20. Mu E, Ding R, An X, Li X, Chen S and Ma X: Heparin attenuates lipopolysaccharide-induced acute lung injury by inhibiting nitric oxide synthase and TGF- $\beta /$ Smad signaling pathway. Thromb Res 129: 479-485, 2012

21. Ni YF, Tian F, Lu ZF, Yang GD, Fu HY, Wang J, Yan XL, Zhao YC, Wang YJ and Jiang T: Protective effect of nicotine on lipopolysaccharide-induced acute lung injury in mice. Respiration 81: 39-46, 2011.

22. Sun Z, Yao XP, Yu WY, et al: Protective effect of auricula polysaccharide on liver function of rats with obstructive jaudice. J Trad Chin Med Pharm 29: 3985-3987, 2014 (In Chinese).

23. Yao XP, Ma Q, Zhang JL, et al: The effects of Auricularia Auricula Extract on NF-xB and SOD in Liver of Rats with Obstructive Jaundice. Res Pract Chin Medicines 27: 35-37, 2013 (In Chinese).

24. Ma Z, Ji W, Fu Q and Ma S: Formononetin inhibited the inflammation of LPS-induced acute lung injury in mice associated with induction of PPAR gamma expression. Inflammation 36: 1560-1566, 2013

25. Torun AN, Kulaksizoglu S, Kulaksizoglu M, Pamuk BO, Isbilen E and Tutuncu NB: Serum total antioxidant status and lipid peroxidation marker malondialdehyde levels in overt and subclinical hypothyroidism. Clin Endocrinol (Oxf) 70: 469-474, 2009. 